\title{
Nano-silver Colloidal Solution Formation by a Simple and Green Method
}

\author{
HAMID REZA GHORBANI* \\ Department of Chemical Engineering, Qaemshahr Branch, \\ Islamic Azad University, Qaemshahr, Iran. \\ *Corresponding author E-mail: hamidghorbani6@gmail.com \\ http://dx.doi.org/10.13005/ojc/300364
}

(Received: June 10, 2014; Accepted: August 05, 2014)

\begin{abstract}
ABSRTACT
Silver nanoparticles were obtained in aqueous medium, at room temperature, by simple and low cost route. The synthesis involves the use of silver nitrate, polyvinylpyrrolidone (pvp), dextrose and water as the silver precursor, stabilizing agent, reducing agent and solvent respectively. In order to identify and analyze nanoparticles, UV - Vis spectroscopy, transitional electron microscopy (TEM), and dynamic light scattering (DLS) were used. All data showed evidence for the formation of silver nanoparticles, with the size of 6-12 nm.
\end{abstract}

Key words: Silver Nanoparticles, Green Synthesis, Dextrose, pvp.

\section{INTRODUCTION}

In recent years, the synthesis and applications of nanoparticles of noble metals have been of a great interest for researchers. Silver nanoparticles provide a wide range of valuable opportunities for such fields of science as catalysis, photonics, photography, chemical sensing, surface enhanced Raman scattering (SERS) and most importantly in the medicinal field as anti-microbial agents $^{1-3}$. These properties extensively depend on the size and shape of the nanomaterial. Synthesis of silver particles is an important topic ${ }^{4}$. Development of methods for nanostructures synthesis is necessary to produce nanoparticles that can be employed in different applications. There are several methods for fabrication of silver nanoparticles. Generally, silver nanoparticles can be prepared by chemical methods such as chemical reduction and electrochemical techniques, physical methods such as Arc-discharge and physical vapor condensation and biological methods such as the use of microorganisms ${ }^{5-8}$.

In 2014, spherical Silver nanoparticles (SNPs) were synthesized by chemical reduction method from a metal precursor silver nitrate in presence of an anionic surfactant and strong reducing agent ${ }^{9}$. In another work, Metallic silver nanoparticles was reduced from silver nitrate by employing the extracellular enzymatic machinery of edible White 
button Mushroom (Agaricus bisporus) ${ }^{10}$.Ghorbani reported biosynthesis of silver nanoparticles using Salmonella typhirium The size of the nanoparticles was determined to be $87 \pm 30 \mathrm{~nm}$, applying dynamic light scattering ${ }^{11}$. In another paper, the Lens culinaris seed extract was used to green synthesis of silver nanoparticles at temperature of $25^{\circ} \mathrm{C}^{12}$. A green low-cost and reproducible yeast mediated synthesis of silver nanoparticles was reported by Jha et al., at room temperature ${ }^{13}$.

This paper presents the synthesis of silver nanoparticles by simple and green route. In this paper, we employed silver nitrate as an initial reagent, dextrose as reducing agent, and polyvinyl pyrrolidone (pvp) as a stabilizer.

\section{MATERIALS AND METHODS}

\section{Materials}

The silver nitrate as the precursor, Dextrose as the reducing agent and polyvinyl pyrrolidone (PVP) as the stabilizing agent to synthesize nano silver colloids were purchased from Sigma-Aldrich.

\section{Preparation}

$1 \mathrm{ml}$ of aqueous solution of dextrose (3 $\mathrm{g} / 100 \mathrm{ml}$ ) and $1 \mathrm{ml}$ polyvinylpyrrolidone $(20 \mathrm{~g} / \mathrm{l})$ were added to $100 \mathrm{ml}$ aqueous solution of silver nitrate $(0.001 \mathrm{M})$. The reaction between this solution and $\mathrm{Ag}+$ ions were carried out in bright conditions for $5 \mathrm{~min}$. After $5 \mathrm{~min}$, the colorless solution of silver nitrate in the container turns into brown color. This color change indicates a possibility of nano-silver colloidal solution production.

\section{Characterization of Silver nanoparticles}

Uv-vis spectroscopy was used to prove the existence of nanoparticles. The morphology and size was determined by the transitional electron microscopy (TEM), and DLS analysis.

\section{RESULTS}

For analytical study of the prepared sample, the amount of absorption within wave length of 350 $-600 \mathrm{~nm}$ was observed by uv-vis spectroscopy. It is known that an absorption band at about 400-420 $\mathrm{nm}$ due to surface plasmon resonance in silver nanoparticles ${ }^{14}$. Fig. 1 shows the UV-Vis spectra of
Nano-silver Colloidal Solution recorded between 350 and $600 \mathrm{~nm}$. As illustrated the SPR band cantered $412 \mathrm{~nm}$ confirms the formation of silver nanoparticles in the solution ${ }^{15}$.

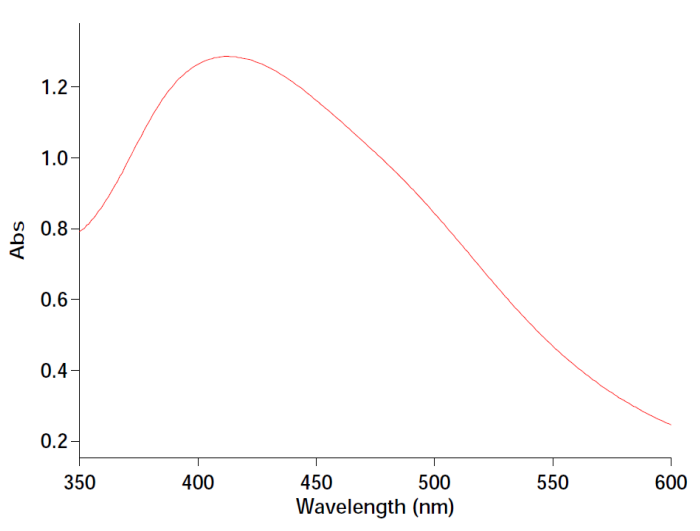

Fig.1: UV-vis spectra of nano-silver colloid

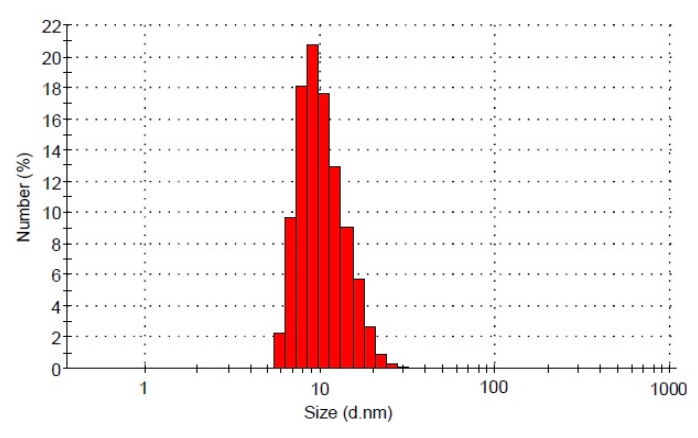

Fig.2. The size distribution of silver nanoparticles by number

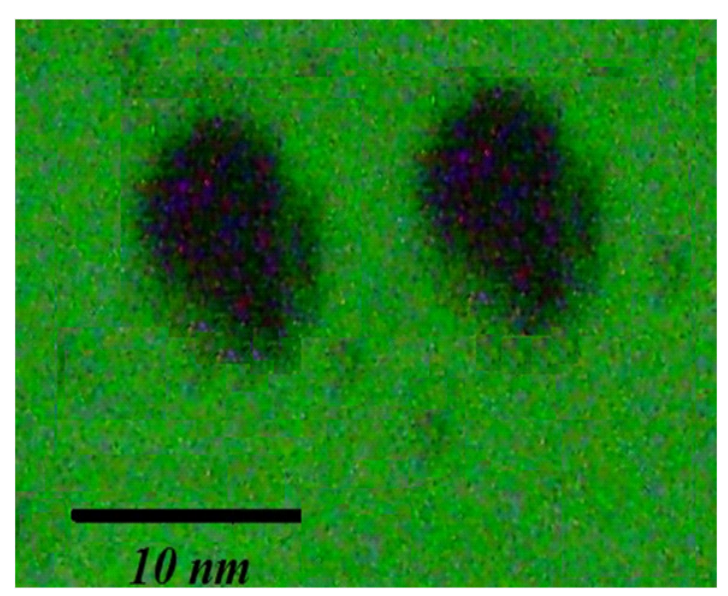

Fig. 3: TEM micrograph of silver nanoparticles produced by dextrose (scale bar $=10 \mathrm{~nm}$ ) 
It is well known that silver can be reduced from $\mathrm{Ag}+$ to $\mathrm{AgO}$ by dextrose. Dynamic light scattering is a widely used technique for the determination of particle size in colloidal solution. As is seen in figure 2, average size of nanoparticle synthesized is $9 \mathrm{~nm}$. The distribution of silver nanoparticles is about $6 \mathrm{~nm}$ which indicates narrow distribution of the nanoparticles. SEM analysis was carried out to understand the topology of silver nanoparticles. At the end, silver nanoparticles were studied by transitional electron microscopy (TEM). Fig. 3 confirms silver nanoparticles formation at nano size.

\section{CONCLUSIONS}

This is an environment friendly one-step method for synthesis of nano-silver colloid. The dextrose acted as a reducing agent of $\mathrm{AgNO}_{3}$ and polyvinylpyrrolidone (pvp) as a capping material. Nanoparticles in the range of $\sim 9 \mathrm{~nm}$ are synthesized by dextrose when it is added to AgNO3. It seems this method is a fast and green method to form Ag nanopartciles. The Ag nanoparticles were characterized by UV-visible, TEM and DLS analysis.

\section{REFERENCES}

1. Prabhu, S.; Poulose, E.K. Int. Nano Lett. 2012, 2, 32.

2. Alahmad, A.; Eleoui, M.; Falah, A.; Alghoraib, I. Phys Sci Res Int., 2013, 1(4), 89-96.

3. Dong, P. V.; Ha, C. H.; Binh, L. T. Int. Nano Lett., 2012, 2, 9.

4. Kasbohm, J. Int J Nanomedicine, 2012, 7, 1543-1550.

5. Lanje1, A. S.; Sharma, S. J.; Pode, R. D. J. Chem. Pharm. Res., 2010, 2(3), 478-483.

6. Mani, U.; Dhanasingh, S.; Arunachalam, R.; Paul, E.; Shanmugam, O.; Rose, C.; Mandal, A. B. Prog Nanotechnol Nanomater, 2013, 2 (1), 21-25.

7. Moazeni, M.; Shahverdi, A. R.; Nabili, M.; Noorbakhsh, F.; Rezaie, S. Nanomedicine Journal, 2014, 1(4), 267-275.

8. Mohan, S.; Oluwafemi, O. S.; George, S. C.; Jayachandran, V. P.; Lewu, F. B.; Songca S. P.; Kalarikkal, N.; Thomas, S. Carbohydr Polym, 2014, 15, 469-474.
9. Ghorbani, H. R.; Safekordi, A. A.; Attar, H.; Rezayat Sorkhabadi, S. M. Chem. Biochem. Eng. Q., 2011, 25 (3,) 317-326.

10. Zambare; A.; Nerpagar, T.; Chaudhari, N.; Manchalwad, P.; Harke, S. Int. J. Nano Dimens., 2014, 5(6), 569-573.

11. Papaiah, S.; Seshadri Goud, T. E.; Devi Prasad, B. S.; Narasimha, G.; Vemana, K. Int. J. Nano Dimens., 2014, 5(2), 139-144.

12. Ghorbani, H.R. J. Nanostructure Chem., 2013, 3, 29.

13. Shams, S.; Pourseyedi, S. H.; Hashemipour Rafsanjani, H. Int. J. Nanosci Nanotechnol, 2014, 10(2), 127-132.

14. Jha; A. K.; Prasad, K.; Kulkarni, A. R. Int. J. Nanosci Nanotechnol, 2008, 4(1), 17-22.

15. Wang, Q.; Mao, P. F. Int. J. Nano Dimens, 2013, 4(2), 167-170.

16. Mohammadinejad; R.; Pourseyedi, S. H.; Baghizadeh, A.; Ranjbar, S. H.; Mansoori, G. A. Int. J. Nanosci Nanotechnol, 2013, 9(4), 221-226. 